\title{
Facile One-Pot Synthesis of Novel Hexahydro-2-quinolinecarboxylic Acids under Solvent-Free Reaction Conditions
}

\author{
Saeed Balalaie $^{{ }^{*}}$, Shahrzad Abdolmohammadi ${ }^{2}$, Bita Soleimanifard ${ }^{\mathbf{1}}$ \\ ${ }^{1}$ Peptide Chemistry Research Center, K.N. Toosi University of Technology, Tehran, Iran \\ ${ }^{2}$ Department of Chemistry, East Tehran Branch (Qiamdasht), Islamic Azad University, Tehran, Iran \\ Email: "balalaie@Kntu.ac.ir
}

Received June 15, 2012; revised July 21, 2012; accepted August 5, 2012

\begin{abstract}
A simple and fastthree-component synthesis of new and biologically active hexahydro-2-quinolinecarboxylic acid scaffold 4 was carried out using cyclocondensation reaction of arylmethylidenepyruvic acids 1, 1,3-cyclohexandiones 2 and ammonium acetate 3 under solvent-free conditions and at room temperature. This protocol has the advantages of facility, easy work-up, high yields, short reaction time and environmentally friendly character.
\end{abstract}

Keywords: Arylmethylidene Pyruvic Acid; Hexahydro-2-quinolinecarboxylic Acids; Solvent-Free Conditions; One-Pot Three-Component Reaction

\section{Introduction}

Heterocyclic containing nitrogen atom in their skeleton, are abundant in nature and exhibit various important chemical and biological activities. Among them quinolones are of interest because they constitute an important class of natural and non-natural products, many of which have different biological activities such as antimalarial[1], antibacterial[2], antimicrobial[3], and antistaphylococcal activities[4]. Meanwhile, an important class of antibiotics possess 4-quinolone framework in their structure [5]. In addition, they can be used as useful drugs for the treatment of Alzheimer's disease [6]. Recently, Kumar et al. showed that some compounds with quinolone skeleton act as a lead in the antidiabetic discovery [7], with effects such as glycologenphosphorylase inhibitors [8-12], or as lipid lowering agent [13] and also for the treatment of metabolic disorder. Also, a number of quinolones are useful in industry as pigments [14].

There are several methods available for the synthesis of quinolinones, for example: 1) the reaction of aromatic aldehydes, dimedone and 3-amino-5-methylpyrazolevia traditional heating in ethanol [15] or in water, 2) the reaction of Schiff base derivatives and dimedone in the presence of TEBAC as catalyst [16]; 3) the condensation reaction of Baylis-Hillman adductive products with cyclic enamine in $n$-buthanol at reflux conditions [17], and condensation of chalcones with dimedone and ammonium

${ }^{*}$ Corresponding author. acetate in the presence of PTSA at reflux condition [7]. However, these methods are still not satisfactory in view of using catalyst, organic solvent waste, harsh reaction conditions, long reaction time and operational complexity. These facts and in continuation our previous studies on the development of new facile routes in heterocyclic synthesis [18-25] led us to explore other clean method for the synthesis of a new class of the important quinolinones derivatives.

The recent focus on the green chemical theme of eliminating the use of solvents also encouraged us to extent our studies to neat reaction conditions [26-34]. In this article, we report a very simple, efficient and clean synthetic route to 5-oxo-4-phenyl-1,4,5,6,7,8-hexahydro-2quinolinecarboxylic acid derivatives via three-component reaction of arylmethylidenepyruvic acids, 1,3-cyclohexandiones and ammonium acetate in solvent-free reaction condition. Moreover, the produced target compounds as heterocyclic $\alpha$-amino carboxylic acids can be of interest for the synthesis of novel peptides containing a heterocyclic moiety in their skeleton, which could lead to some biologically active compounds. On the other hand, these compounds can be used as an efficient starting material for further multicomponent reactions, because of the possession of both amine and carboxylic acid functional groups.

In this reaction, arylmethylidenepyruvic acid plays an important role as an attractive starting material, for several reasons such as: 1) higher reactivity in comparison to 
usual $\alpha, \beta$-unsaturated ketones; 2) containing active functional groups which can be used for further synthesis, and 3) easy preparation, i.e., they were synthesized according to the known procedure [35] by reaction of aromatic aldehydes and pyruvic acid in an aqueous $\mathrm{MeOH}$ solution of $\mathrm{KOH}$.

\section{Results and Discussion}

When the reaction of arylmethylidenepyruvic acids 1 , 1,3-cyclohexandiones 2 and ammonium acetate 3 was performed by abrasion in a mortar without using any solvent or catalyst at room temperature, high yields of 5-oxo-4-phenyl-1,4,5,6,7,8-hexahydro-2-quinolinecarbox ylic acid derivatives were obtained (Scheme 1).

In order to apply this reaction to a library synthesis, various kinds of arylmethylidenepyruvic acids and 1,3cyclohexandiones were subjected to give the corresponding 5-oxo-4-phenyl-1,4,5,6,7,8-hexahydro-2-quinolinecarboxylic acids 4 , and representative examples are shown in Table 1.

The structures of compounds $4 \mathrm{a}$ - $4 \mathrm{f}$ were deduced from their spectroscopic data, which displayed in each case, the molecular ion peak at the appropriate $m / z$ values. All of the products exhibited a singlet in ${ }^{1} \mathrm{H}-\mathrm{NMR}$ at about $\delta=4.49-4.59$ ppm for $\mathrm{H}-4$ and also a distinguished peak at 36.2 - $36.8 \mathrm{ppm}$ for C-4 in ${ }^{13} \mathrm{C}-\mathrm{NMR}$ spectroscopy. Meanwhile, ${ }^{1} \mathrm{H}-\mathrm{NMR}$ spectra of compounds $4 \mathrm{~d}-\mathrm{f}$ shows two doublets at $2.20-2.40 \mathrm{ppm}$ with $J=16 \mathrm{~Hz}$ that is related to geminal coupling of H-6 protons. Selected spectroscopic data are reported.
Although we have not yet established the mechanism, a possible explanation is given in Scheme 2. Based on this mechanism, we suggest that, the formation of enamine intermediate 4 is occured in the first step by the reaction of 1,3-cyclohexandione 2 and ammonium acetate 3 , and due to the $C$-nucleophilic character of the enamine intermediate 4 , it is reasonable to assume that 5 can be formed via the initial Michael addition between arylmethylidenepyruvic acids 1 and enamine intermediate 4 to generate the Michael adduct 5, which isomerized under the reaction conditions to yield 6 . Intramolecular cyclization of 6 gives 3 after dehydration of intermediate 7 .

The products are unusual amino acids which could be used for the synthesis of novel peptides with heterocyclic skeleton.

\section{Conclusion}

In conclusion, we have found that the abrasion of arylmethylidenepyruvic acids, 1,3-cyclohexandiones and ammonium acetate leads to a facile synthesis of 5-oxo-4phenyl-1,4,5,6,7,8-hexahydro-2-quinolinecarboxylic acids without the addition of any catalysts under solventfree conditions at room temperature. Besides the simplicity of the method, our synthesized heterocyclic molecules have the potential to be biologically active or used as precursor to produce other biological active compounds. Further investigation about using of these products for the synthesis of novel peptides is going on our laboratory.<smiles>COc1cc(OC(=O)C(C)=O)ccc1C(=O)O</smiles>

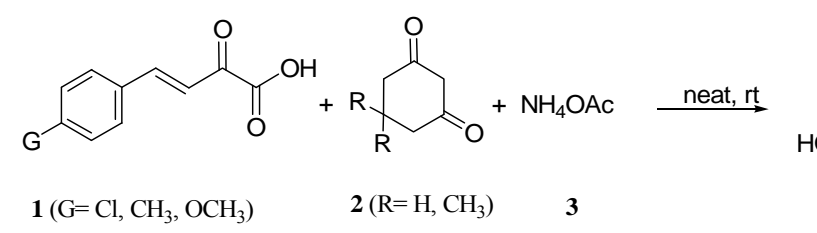<smiles>[R7]C1CC(=O)C2=C(CC(=O)[NH+](C(=O)O)C2)[C]1c1ccc(Cl)cc1</smiles>

Scheme 1. Efficient synthesis of arylmethylidene pyruvic acid 1 and hexahydro-2-quinolinecarboxylic acid $4 a-4 f$.

Table 1. Synthesis of -oxo-4-phenyl-1,4,5,6,7,8-hexahydro-2-quinolinecarboxylic acids 4a - 4f in solvent-free conditions.

\begin{tabular}{ccccc}
\hline Product & $\mathrm{G}$ & $\mathrm{R}$ & M.p. $\left({ }^{\circ} \mathrm{C}\right)$ & Yield $^{\mathrm{a}}(\%)$ \\
\hline $\mathbf{4 a}$ & $\mathrm{Cl}$ & $\mathrm{H}$ & $213-215$ & 95 \\
$\mathbf{4 b}$ & $\mathrm{MeO}$ & $\mathrm{H}$ & $239-241$ & 92 \\
$\mathbf{4 c}$ & $\mathrm{Me}$ & $\mathrm{H}$ & $253-255$ & 93 \\
$\mathbf{4 d}$ & $\mathrm{Cl}$ & $\mathrm{Me}$ & $250-252$ & 98 \\
$\mathbf{4 e}$ & $\mathrm{MeO}$ & $\mathrm{Me}$ & $248-250$ & 95 \\
$\mathbf{4 f}$ & $\mathrm{Me}$ & $\mathrm{Me}$ & $259-261$ & 94 \\
\hline
\end{tabular}

${ }^{\mathrm{a}}$ Yields refer to pure isolated products characterized by IR, ${ }^{1} \mathrm{H}$ - and ${ }^{13} \mathrm{C}-\mathrm{NMR}$ spectroscopy and mass spectrometery. 
<smiles>[R]C1([R])CC(=O)CC(=O)C1</smiles><smiles>[R]C1([R])CC(=N)C(C(CC(=O)C(=O)O)c2ccc(Cl)cc2)C(=O)C1</smiles><smiles>[R]CCC(CC(=O)C(=O)O)c1ccc(O)cc1</smiles><smiles>[R]Cc1c(C(=O)O)cc(C(=O)O)c2c1CC([R])CC2=O</smiles>

3<smiles>[R]C1([R])CC(=O)C2=C(C1)NC(O)(C(=O)O)CC2c1ccc(Cl)cc1</smiles>

Scheme 2. The proposed mechanism for the synthesis of 5-oxo-4-phenyl-1,4,5,6,7,8-hexahydro-2-quinolinecarboxylic acids in solvent-free conditions.

\section{Experimental Section}

Material and methods. Melting points were determined on an Electrothermal 9100 apparatus and were uncorrected. IR spectra were obtained on an ABB FT-IR (FTLA 2000) spectrometer. ${ }^{1} \mathrm{H}$ - and ${ }^{13} \mathrm{C}-\mathrm{NMR}$ spectra were recorded on a BrukerDRX-300 AVANCE at 300 and $75 \mathrm{MHz}$, respectively, using TMS as internal standard and $\operatorname{DMSO}\left(d_{6}\right)$ as solvent. Mass spectrawere obtained using a GC-MS Hewlett Packard (EI, $70 \mathrm{eV}$ ) instrument. The purity of prepared compounds was tested by the elemental analysis of $\mathrm{C}, \mathrm{H}$, and $\mathrm{N}$ elements using a Heraus $\mathrm{CHN}$ rapid analyzer.

General procedure for the preparation of compounds 4a - 4f: A mixture of the arylmethylidenepyruvic acids (1, $1 \mathrm{mmol}), 1,3$-cyclohexandiones $(2,1 \mathrm{mmol})$ and ammo- nium acetate $3(0.144 \mathrm{mg}, 2 \mathrm{mmol})$ was abrade in a mortar for $1.5 \mathrm{~h}$. After completion the reaction which monitored by TLC (ethanol: ethyl acetate, 1:1) the reaction mixture was resolved in a required mixture of $\mathrm{H}_{2} \mathrm{O}$ : EtOH ( in a ratio of $1: 1$ ). After the evaporation of $\mathrm{EtOH}$, the $\mathrm{pH}$ of solution was reached to 2.5 by adding concentrated solution of $\mathrm{HCl}$, the precipitate was filtered and washed with water and $\mathrm{CHCl}_{3}$ respectively to afford the pure products 4 in high yields( $92 \%$ - 98\%).

Selected data for compounds $4 \mathrm{a}$ - 4f:

5-oxo-4-(4-chlorophenyl)-1,4,5,6,7,8-hexahydro-2-q uinolinecarboxylic acid (4a): Yield: $289 \mathrm{mg}$ (95\%). White powder. M.p. $213^{\circ} \mathrm{C}-215^{\circ} \mathrm{C}$. IR (KBr): 3326 , 1690, 1654, $1567 \mathrm{~cm}^{-1}$. ${ }^{1} \mathrm{H}-\mathrm{NMR}\left(300 \mathrm{MHz}, \mathrm{DMSO}-d_{6}\right)$ : $\delta=1.84(\mathrm{~m}, 2 \mathrm{H}, \mathrm{H}-7), 2.16(\mathrm{~m}, 2 \mathrm{H}, \mathrm{H}-8), 2.43(\mathrm{~m}, 1 \mathrm{H}$, H-6), 2.67 (m, 1 H, H-6'), 4.59 (d, $J=5.6 \mathrm{~Hz}, 1 \mathrm{H}, \mathrm{H}-4)$, $5.90(\mathrm{~d}, J=4.8 \mathrm{~Hz}, 1 \mathrm{H}, \mathrm{H}-3), 7.17$ (d, $J=8.3 \mathrm{~Hz}, 2 \mathrm{H}$, H-Ar), 7.30 (d, J=8.3 Hz, 2 H, H-Ar), 8.70 (s, $1 \mathrm{H}, \mathrm{NH}$ ), 13.27 (brs, $1 \mathrm{H}, \mathrm{COOH})$ ppm. ${ }^{13} \mathrm{C}-\mathrm{NMR}(75 \mathrm{MHz}$, DMSO- $\left.d_{6}\right): \delta=20.8,26.6,36.4,36.8,105.6,115.4$, $127.1,128.2,129.1,130.5,145.8,154.3,163.6,194.3$ ppm. MS (EI $70 \mathrm{eV}): m / z(\%): 305(\mathrm{M}+2,32), 303\left(\mathrm{M}^{+}\right.$, 55), 285 (32), 257 (100), 192 (85), 174 (61), 146 (93). Anal.Calcd.for $\mathrm{C}_{16} \mathrm{H}_{14} \mathrm{NO}_{3} \mathrm{Cl}$ (303.74): C, 63.27; H, 4.65; N, 4.61. Found: C, 63.20; H, 4.62; N, 4.57.

5-oxo-4-(4-methoxyphenyl)-1,4,5,6,7,8-hexahydro-2 -quinolinecarboxylic acid (4b): Yield: $275 \mathrm{mg}(92 \%)$. White powder. M.p. $239^{\circ} \mathrm{C}-241^{\circ} \mathrm{C}$. IR (KBr): 3319 , $1695,1570,1510 \mathrm{~cm}^{-1} .{ }^{1} \mathrm{H}-\mathrm{NMR}\left(300 \mathrm{MHz}, \mathrm{DMSO}-d_{6}\right)$ : $\delta=1.81(\mathrm{~m}, 2 \mathrm{H}, \mathrm{H}-7), 2.17(\mathrm{~m}, 2 \mathrm{H}, \mathrm{H}-8), 2.46(\mathrm{~m}, 1 \mathrm{H}$, H-6), 2.63 (m, 1 H, H-6'), 3.68 (s, 3 H, OCH ${ }_{3}$ ), 4.52 (d, J $=5.6 \mathrm{~Hz}, 1 \mathrm{H}, \mathrm{H}-4), 5.91(\mathrm{dd}, J=5.7,1.5 \mathrm{~Hz}, 1 \mathrm{H}, \mathrm{H}-3)$, 6.80 (d, $J=8.6 \mathrm{~Hz}, 2 \mathrm{H}, \mathrm{H}-\mathrm{Ar}), 7.06$ (d, $J=8.6 \mathrm{~Hz}, 2 \mathrm{H}$, H-Ar), 8.58 (s, 1 H, NH), 13.00 (brs, $1 \mathrm{H}, \mathrm{COOH}$ ) ppm. ${ }^{13} \mathrm{C}-\mathrm{NMR}\left(75 \mathrm{MHz}, \mathrm{DMSO}-d_{6}\right): \delta=20.9,26.6,35.9$, $36.8,55.0,106.2,113.6,116.0,126.8,128.2,139.3$, 153.8, 157.5, 163.9, 194.3 ppm. MS (EI $70 \mathrm{eV}): \mathrm{m} / z(\%)$ : $299\left(\mathrm{M}^{+}, 19\right), 273$ (100), 253 (79), 217 (87), 161 (60). 
Anal.Calcd.for $\mathrm{C}_{17} \mathrm{H}_{17} \mathrm{NO}_{4}$ (299.33): C, 68.22; H, 5.72; N, 4.68. Found: C, 68.18; H, 5.68; N, 4.71.

5-oxo-4-(4-methylphenyl)-1,4,5,6,7,8-hexahydro-2-q uinolinecarboxylic acid (4c): Yield: $263 \mathrm{mg}$ (93\%). White powder. M.p. $253^{\circ} \mathrm{C}-255^{\circ} \mathrm{C}$. IR (KBr): 3322 , $1693,1654,1568 \mathrm{~cm}^{-1} .{ }^{1} \mathrm{H}-\mathrm{NMR}\left(300 \mathrm{MHz}\right.$, DMSO- $\left.d_{6}\right)$ : $\delta=1.83(\mathrm{~m}, 2 \mathrm{H}, \mathrm{H}-7), 2.15(\mathrm{~m}, 2 \mathrm{H}, \mathrm{H}-8), 2.21(\mathrm{~s}, 3 \mathrm{H}$, $\mathrm{CH}_{3}$ ), 2.45 (m, $\left.1 \mathrm{H}, \mathrm{H}-6\right), 2.66$ (m, $1 \mathrm{H}, \mathrm{H}-6$ ') 4.53 (d, $J$ $=5.6 \mathrm{~Hz}, 1 \mathrm{H}, \mathrm{H}-4), 5.90(\mathrm{~d}, J=4.9 \mathrm{~Hz}, 1 \mathrm{H}, \mathrm{H}-3), 7.03$ (s, $4 \mathrm{H}, \mathrm{H}-\mathrm{Ar}), 8.60$ (s, $1 \mathrm{H}, \mathrm{NH}), 13.00$ (brs, $1 \mathrm{H}, \mathrm{COOH})$ ppm. ${ }^{13} \mathrm{C}-\mathrm{NMR}\left(75 \mathrm{MHz}\right.$, DMSO- $\left.d_{6}\right): \delta=20.6,20.9$, 26.6, 36.4, 36.8, 106.0, 116.2, 126.7, 127.2, 128.8, 134.9, 144.0, 153.9, 163.7, $194.3 \mathrm{ppm}$. MS (EI $70 \mathrm{eV}): \mathrm{m} / z(\%)$ : 283 (M+1,90), 265 (22), 237 (100), 192 (88), 174 (67), 146 (93). Anal.Calcd.for $\mathrm{C}_{17} \mathrm{H}_{17} \mathrm{NO}_{3}$ (283.33): C, 72.07; H, 6.05; N, 4.94. Found: C, 72.01; H, 6.02; N, 4.87.

7,7-dimethyl-5-oxo-4-(4-chlorophenyl)-1,4,5,6,7,8-h exahydro-2-quinolinecarboxylic acid (4d): Yield: 325 $\mathrm{mg}(98 \%)$. White powder. M.p. $250^{\circ} \mathrm{C}-252^{\circ} \mathrm{C}$. IR (KBr): 3375, 1701, 1663, $1564 \mathrm{~cm}^{-1}$. ${ }^{1} \mathrm{H}-\mathrm{NMR}(300 \mathrm{MHz}$, DMSO- $\left.d_{6}\right): \delta=0.91\left(\mathrm{~s}, 3 \mathrm{H}, \mathrm{CH}_{3}\right), 0.98\left(\mathrm{~s}, 3 \mathrm{H}, \mathrm{CH}_{3}\right)$, $1.96(\mathrm{~d}, J=16.1 \mathrm{~Hz}, 1 \mathrm{H}, \mathrm{H}-6), 2.13(\mathrm{~d}, J=16.1 \mathrm{~Hz}, 1 \mathrm{H}$, H-6'), 2.46 (m, $2 \mathrm{H}, \mathrm{H}-8), 4.57$ (d, $J=5.5 \mathrm{~Hz}, 1 \mathrm{H}, \mathrm{H}-4)$, $5.88(\mathrm{~d}, J=5.5 \mathrm{~Hz}, 1 \mathrm{H}, \mathrm{H}-3), 7.17$ (d, $J=8.3 \mathrm{~Hz}, 2 \mathrm{H}$, $\mathrm{H}-\mathrm{Ar}$ ), 7.30 (d, $J=8.3 \mathrm{~Hz}, 2 \mathrm{H}, \mathrm{H}-\mathrm{Ar}), 8.64$ (s, $1 \mathrm{H}, \mathrm{NH})$, 13.28 (brs, $1 \mathrm{H}, \mathrm{COOH})$ ppm. ${ }^{13} \mathrm{C}-\mathrm{NMR}(75 \mathrm{MHz}$, DMSO- $\left.d_{6}\right): \delta=27.0,29.0,31.9,36.7,50.3,104.7,115.4$, 127.2, 128.2, 129.2, 130.5, 146.0, 152.4, 163.7, 193.9 ppm. MS (EI $70 \mathrm{eV}): m / z(\%): 333(\mathrm{M}+2,22), 331\left(\mathrm{M}^{+}\right.$, 60), 313 (13), 285 (100), 220 (95), 202 (47), 174 (94). Anal.Calcd.for $\mathrm{C}_{18} \mathrm{H}_{18} \mathrm{NO}_{3} \mathrm{Cl}$ (331.80): C, 65.16; H, 5.47; N, 4.22. Found: C, 65.19; H, 5.51; N, 4.26.

7,7-dimethyl-5-oxo-4-(4-methoxyphenyl)-1,4,5,6,7,8 -hexahydro-2-quinolinecarboxylic acid (4e): Yield: $311 \mathrm{mg}(95 \%)$. White powder. M.p. $248^{\circ} \mathrm{C}-250^{\circ} \mathrm{C}$. IR (KBr): $3369,1701,1661,1565 \mathrm{~cm}^{-1} .{ }^{1} \mathrm{H}-\mathrm{NMR}(300 \mathrm{MHz}$, DMSO- $\left.d_{6}\right): \delta=0.92\left(\mathrm{~s}, 3 \mathrm{H}, \mathrm{CH}_{3}\right), 0.98\left(\mathrm{~s}, 3 \mathrm{H}, \mathrm{CH}_{3}\right)$, $1.96(\mathrm{~d}, J=16.1 \mathrm{~Hz}, 1 \mathrm{H}, \mathrm{H}-6), 2.12$ (d, $J=16.1 \mathrm{~Hz}, 1 \mathrm{H}$, H-6'), 2.45 (m, 2 H, H-8), 3.68 (s, 3 H, $\mathrm{OCH}_{3}$ ), 4.49 (d, $J$ $=5.6 \mathrm{~Hz}, 1 \mathrm{H}, \mathrm{H}-4), 5.88(\mathrm{~d}, J=5.6 \mathrm{~Hz}, 1 \mathrm{H}, \mathrm{H}-3), 6.80$ (d, $J=8.5 \mathrm{~Hz}, 2 \mathrm{H}, \mathrm{H}-\mathrm{Ar}), 7.06(\mathrm{~d}, J=8.5 \mathrm{~Hz}, 2 \mathrm{H}$, H-Ar), 8.53 (s, $1 \mathrm{H}, \mathrm{NH}), 13.00$ (brs, $1 \mathrm{H}, \mathrm{COOH}) \mathrm{ppm}$. ${ }^{13} \mathrm{C}-\mathrm{NMR}\left(75 \mathrm{MHz}, \mathrm{DMSO}-d_{6}\right): \delta=26.9,29.1,31.9$, $36.2,50.4,55.0,105.2,113.6,116.3,126.6,128.3,139.5$, 152.0, 157.6, 163.8, 193.9 ppm. MS (EI $70 \mathrm{eV}): \mathrm{m} / \mathrm{z}(\%)$ : $327\left(\mathrm{M}^{+}, 71\right), 309$ (6), 281 (100), 220 (54), 202 (24), 174 (67). Anal.Calcd.for $\mathrm{C}_{19} \mathrm{H}_{21} \mathrm{NO}_{4}$ (321.38): C, 69.71; H, 6.47; N, 4.36. Found: C, 69.68; H, 6.50; N, 4.32.

7,7-dimethyl-5-oxo-4-(4-methylphenyl)-1,4,5,6,7,8-h exahydro-2-quinolinecarboxylic acid (4f): Yield: 292 $\mathrm{mg}(94 \%)$. White powder. M.p. $259^{\circ} \mathrm{C}-261^{\circ} \mathrm{C}$. IR (KBr): 3369, 1702, 1661, $1564 \mathrm{~cm}^{-1}$. ${ }^{1} \mathrm{H}-\mathrm{NMR}$ (300 MHz, DMSO- $\left.d_{6}\right): \delta=0.92\left(\mathrm{~s}, 3 \mathrm{H}, \mathrm{CH}_{3}\right), 0.98\left(\mathrm{~s}, 3 \mathrm{H}, \mathrm{CH}_{3}\right)$, 1.95 (d, $J=16.0 \mathrm{~Hz}, 1 \mathrm{H}, \mathrm{H}-6), 2.11$ (d, $J=16.0 \mathrm{~Hz}, 1 \mathrm{H}$,
H-6' ), 2.21 (s, 3 H, $\mathrm{CH}_{3}$ ), 2.45 (m, $2 \mathrm{H}, \mathrm{H}-8$ ), 4.50 (d, J $=5.2 \mathrm{~Hz}, 1 \mathrm{H}, \mathrm{H}-4), 5.88(\mathrm{~d}, J=4.8 \mathrm{~Hz}, 1 \mathrm{H}, \mathrm{H}-3), 7.03$ (s, $4 \mathrm{H}, \mathrm{H}-\mathrm{Ar}), 8.51$ (s, $1 \mathrm{H}, \mathrm{NH}), 12.95$ (brs, $1 \mathrm{H}, \mathrm{COOH})$ ppm. ${ }^{13} \mathrm{C}-\mathrm{NMR}\left(75 \mathrm{MHz}, \mathrm{DMSO}-d_{6}\right): \delta=20.6,26.9$, $29.1,31.9,36.8,50.3,105.0,116.2,126.8,127.3,128.8$, 135.0, 144.3, 152.1, 163.8, 193.8 ppm. MS (EI $70 \mathrm{eV})$ : m/z (\%): 311 (M $\left.\mathrm{M}^{+}, 67\right), 293$ (7), 265 (100), 220 (76), 202 (30), 174 (71). Anal.Calcd.for $\mathrm{C}_{19} \mathrm{H}_{21} \mathrm{NO}_{3}$ (311.38): C, 73.29; H, 6.80; N, 4.50. Found: C, 73.34; H, 6.82; N, 4.56 .

\section{Graphical Abstract}

Facile One-Pot Synthesis of Novel Hexahydro-2-quinolinecarboxylic Acids, under Solvent-Free Reaction conditions.

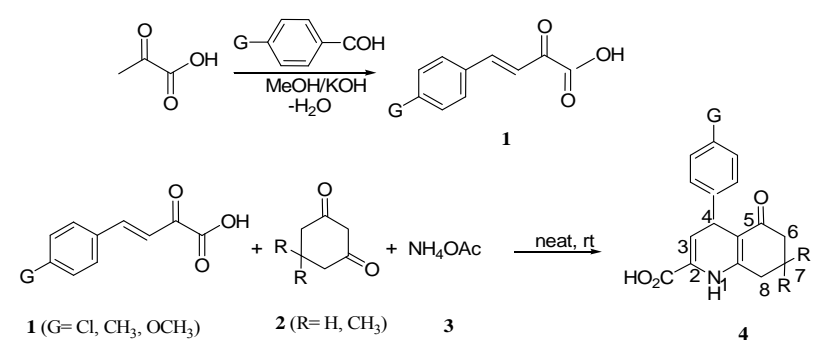

\section{Acknowledgements}

S. B. gratefully acknowledges Alexander von Humboldt foundation for their research fellowship. We are also grateful to Prof. R. Gleiter for fruitful and helpful discussions. We also wish to express our gratitude to Mr. M. Jalilevand, managing director of Kimia Exir Company for providing us with donations of chemicals and financial support.

\section{REFERENCES}

[1] J. A. Joule, K. Mills and G. F. Smith, "Heterocyclic Chemistry," 3rd Edition, Chapman \& Hall, London, 1995.

[2] M. Q. Zhang, A. Haemers, D. Vanden Berghe, S. R. Pattyn, W. Bollaert and I. Levshin, "Quinolone Antibacterials. 2. 6-Substituted-7-(2-thiazolyl and thiazolidinyl) Quinolones," Journal of Heterocyclic Chemistry, Vol. 28, No. 3, 1991, pp. 1685-1695. doi:10.1002/jhet.5570280324

[3] K. Roy, R. P. Srivastava, B. L. Tekwani, V. C. Pandev and A. P. Bhaduri, "Syntheses and Exploration of New Biological Activities in Ethyl 6/7-Substituted and 6,7Disubstituted Quinolin-4-one-3-carboxylates," Bioorganic \& Medicinal Chemistry Letters, Vol. 6, No. 2, 1996, pp. 1121-1126. doi:10.1016/0960-894X(95)00577-G

[4] M. Reuman, S. J. Daum, B. Singh, M. P. Wentland, R. B. Perni, P. Pennock, P. M. Carabateas, M. D. Gruett, M. T. Saindane, P. H. Dorff, S. A. Coughlin, D. M. Sedlock, J. B. Rake and G. Y. Lesher, "Synthesis and Antibacterial Activity of Some Novel 1-Substituted 1,4-Dihydro-4-oxo- 
7-pyridinyl-3-quinolinecarboxylic Acids. Potent An- tistaphylococcal Agents," Medicinal Chemistry, Vol. 38, No. 14, 1995, pp. 2531-2540. doi:10.1021/jm00014a005

[5] S. Bogialli, G. D'Ascenzo, A. Di Corcia, A. Laganà and S. Nicolardi, "A Simple and Rapid Assay Based on Hot Water Extraction and liquid Chromatography-Tandem Mass Spectrometry for Monitoring Quinolone Residues in Bovine Milk," Food Chemistry, Vol. 108, No. 1, 2008, pp. 2354-2360. doi:10.1016/j.foodchem.2007.10.044

[6] W. H. Suh, K. S. Suslick and Y. H. Suh, "Therapeutic Agents for Alzheimer's Disease," Current Medicinal Chemistry Central Nervous System Agents, Vol. 5, No. 4, 2005, pp. 259-269. doi:10.2174/156801505774913035

[7] A. Kumar, S. Sharma, V. D. Tripathi, R. A. Maurya, S. P. Srivastava, G. Bhatia, A. K. Tamrakar and A. K. Srivastava, "Design and Synthesis of 2,4-Disubstituted Polyhydroquinolines as Prospective Antihyperglycemic and Lipid Modulating Agents," Bioorganic \& Medicinal Chemistry Letters, Vol. 18, No. 11, 2010, pp. 4138-4148. doi:10.1016/j.bmc.2009.11.061

[8] N. Bergans, W. Stalmans, S. Goldmann and F. Vanstapel, "Molecular Mode of Inhibition of Glycogenolysis in Rat Liver by the dihydropyridine Derivative, BAY R3401: Inhibition and Inactivition of Glycogen Phosphorylase by an Activated Metabolite," Diabetes, Vol. 49, No. 9, 2000, pp. 1419-1426. doi:10.2337/diabetes.49.9.1419

[9] S. Goldmann, H. J. Ahr, W. Puls, H. Bischoff, D. Petzinna, K. Schlossmann and J. Bender, "Dihydropyridine Compounds and Their Use in Reducing Blood Sugar," Patent No. 4786641, 1988.

[10] G. H. Ladouceur, J. H. Cook, E. M. Doherty, W. R. Schoen, M. L. MacDougall and J. N. Livingston, "Discovery of 5-Hydroxyalkyl-4-phenylpyridines as a New Class of Glucagon Receptor Antagonists," Bioorganic \& Medicinal Chemistry Letters, Vol. 12, No. 3, 2002, pp. 2461-2464. doi:10.1016/S0960-894X(01)00766-1

[11] N. G. Oikonomakos, K. E. Tsitsanou, S. E. Zographos, V. T. Skamnaki, S. Goldmann and H. Bischoff, "Allosteric Inhibition of Glycogen Phosphorylase a by the Potential Antidiabetic Drug 3-Isopropyl 4-(2-Chlorophenyl)-1,4di-hydro-1-ethyl-2-methyl-pyridine-3,5,6-tricarboxylate," Protein Science, Vol. 8, No. 10, 1999, pp. 1930-1945. doi:10.1110/ps.8.10.1930

[12] W. R. Schoen, G. H. Ladouceur, J. H. Cook, T. G. Lease, D. J. Wolanin, R. H. Kramss, D. L. Hertzog and M. H. Osterhout, "Substituted biphenyls," Patent No. 6218431, 2001.

[13] G. S. Poindexter, R. T. Swann, M. A. Bruce, M. A. Morton, Y. Huang, S. Y. Sit and J. G. Breitenbucher, "Dihydropyridine NPY Antagonists: Cyanoguanidine Derivatives," Patent No. 6001836, 1999.

[14] E. Q. Adams, H. L. Haller, "Kryptocyanines. A New Series of Photosensitizing Dyes," Journal of the American Chemical Society, Vol. 42, No. 12, 1920, pp. 26612663. doi:10.1021/ja01457a026

[15] J. Quiroga, D. Mejía, B. Insuasty, R. Abonía, M. Nogueras, A. Sánchez, J. Cobo and J. N. Low, "Regioselective Synthesis of 4,7,8,9-Tetrahydro-2H-pyrazolo[3,4-b]quinolin-5(6H)-ones. Mechanism and Structural Analy- sis," Tetrahedron, Vol. 57, No. 32, 2001, pp. 6947-6953. doi:10.1016/S0040-4020(01)00649-4

[16] X.-S. Wang, M.-M. Zhang, Z.-S. Zeng, D.-Q. Shi, S.-J. Tu, X.-Y. Wei and Z.-M. Zong, "A Simple and Clean Procedure for the Synthesis of Polyhydroacridine and Quinoline Derivatives: Reaction of Schiff Base with 1,3Dicarbonyl Compounds in Aqueous Medium," Tetrahedron Letters, Vol. 46, No. 42, 2005, pp. 7169-7173. doi:10.1016/j.tetlet.2005.08.091

[17] S. Gowrisankar, J. E. Na, M. J. Lee and J. N. Kim, "Synthesis of 3-Benzyl-2-hydroxy-7,8-dihydro-6H-quinolin-5 -ones from Baylis-Hillman Adducts," Cheminform, Vol. 36, No. 26, 2005, pp. 319-322. doi:10.1002/chin.200526083

[18] S. Abdolmohammadi and S. Balalaie, "Novel and Efficient Catalysts for the One-Pot Synthesis of 3,4-Dihydropyrano[c]chromene Derivatives in Aqueous Media," Tetrahedron Letters, Vol. 48, No. 18, 2007, pp. 3299-3303. doi:10.1016/j.tetlet.2007.02.135

[19] S. Balalaie, S. Abdolmohammadi and B. Soleimanifard, "An Efficient Synthesis of Novel Hexahydropyrido[2,3d]pyrimidine Derivatives from (Arylmethylidene)pyruvic Acids (=(3E)-4-Aryl-2-oxobut-3-enoic Acids) in Aqueous Media," Helvetica Chimica Acta, Vol. 92, No. 5, 2009, pp. 2932-2936. doi:10.1002/hlca.200800318

[20] S. Balalaie, M. Bararjanian, S. Hosseinzadeh, F. Rominger, H. R. Bijanzadeh and E. Wolf, "Designing a Sequential Ugi/Ullmann Type Reaction for the Synthesis of Indolo[1,2-a]quinoxalinones Catalyzed by CuI/1-Proline," Tetrahedron, Vol. 67, No. 38, 2011, pp. 7294-7300. doi:10.1016/j.tet.2011.07.052

[21] M. Bararjanian, S. Balalaie, F. Rominger and S. Barouti, "A Novel and Efficient One-Pot Synthesis of 2-Aminopyrimidinones and Their Self-Assembly," Helvetica Chimica Acta, Vol. 93, No. 4, 2010, pp. 2777-2784. doi:10.1002/hlca.200900319

[22] M. Bararjanian, S. Balalaie, F. Rominger, B. Movassagh and H. R. Bijanzadeh, "Six-Component Reactions for the Stereoselective Synthesis of 3-Arylidene-2-oxindoles via Sequential One-Pot Ugi/Heck Carbocyclization/Sonogashira/Nucleophilic Addition," The Journal of Organic Chemistry, Vol. 75, No. 9, 2010, pp. 2806-2812. doi:10.1021/j0902713x

[23] M. Bararjanian, S. Hosseinzadeh, S. Balalaie and H. R. Bijanzadeh, "Palladium Catalyzed Stereoselective Synthesis of 3-(Anilinoarylmethylene)-2-oxindoles as Hesperadin Analogoues," Tetrahedron, Vol. 67, No. 14, 2011, pp. 2644-2650. doi:10.1016/j.tet.2011.02.005

[24] M. J. Khoshkholgh, S. Balalaie, R. Gleiter and F. Rominger, "Intramolecular Hetero-Diels-Alder Reaction of 1-Oxa-1,3-butadienes with Terminal Acetylenes in Aqueous Media Using CuI," Tetrahedron, Vol. 64, No. 48, 2008, pp. 10924-10929. doi:10.1016/j.tet.2008.08.056

[25] M. J. Khoshkholgh, M. Lotfi, S. Balalaie and F. Rominger, "Efficient Synthesis of Pyrano[2,3-c]coumarins via Intramolecular Domino Knoevenagel Hetero-Diels-Alder Reactions," Tetrahedron, Vol. 65, No. 21, 2009, pp. 42284234. doi:10.1016/j.tet.2009.03.032

[26] G. Choudhary and R. K. Peddinti, "An Expeditious, 
Highly Efficient, Catalyst-Free and Solvent-Free Synthesis of Nitroamines and Nitrosulfides by Michael Addition," Green Chemistry, Vol. 13, No. 2, 2011, pp. 22762282. doi:10.1039/c0gc00830c

[27] Y. Gu, R. De Sousa, G. Frapper, C. Bachmann, J. Barrault and F. Jerome, "Catalyst-Free Aqueous Multicomponent Domino Reactions from Formaldehyde and 1,3Dicarbonyl Derivatives, "Green Chemistry, Vol. 11, No. 12, 2009, pp. 1968-1972. doi:10.1039/b913846c

[28] S. Iliescu, G. Ilia, N. Plesu, A. Popa and A. Pascariu, "Solvent and Catalyst-Free Synthesis of Polyphosphates," Green Chemistry, Vol. 8, No. 8, 2006, pp. 727-730. doi:10.1039/b602462a

[29] S. L. Jain, S. Singhal and B. Sain, "PEG-Assisted Solvent and Catalyst Free Synthesis of 3,4-Dihydropyrimidinones under Mild Reaction Conditions," Green Chemistry, Vol. 9, No. 7, 2007, pp. 2740-2741. doi:10.1039/b702311a

[30] M. A. P. Martins, C. P. Frizzo, D. N. Moreira, L. Buriol and P. Machado, "Solvent-Free Heterocyclic Synthesis," Chemical Reviews, Vol. 109, No. 9, 2009, pp. 4140-4182. doi:10.1021/cr9001098

[31] B. C. Ranu, S. S. Dey and A. Hajra, "Highly Efficient Acylation of Alcohols, Amines and Thiols under Solvent-Free and Catalyst-Free Conditions,"Green Chemis- try, Vol. 5, No. 1, 2003, pp. 44-46. doi:10.1039/b211238h

[32] L. D. S. Yadav, S. Singh and V. K. Rai, "Catalyst-Free, Step and Pot Economic, Efficient Mercaptoacetylative Cyclisation in $\mathrm{H}_{2} \mathrm{O}$ : Synthesis of 3-Mercaptocoumarins," Green Chemistry, Vol. 11, No. 6, 2009, pp. 878-882. doi:10.1039/b904655k

[33] S. Yan, Y. Chen, L. Liu, N. He and J. Lin, "Three-Component Solvent-Free Synthesis of Highly Substituted Bicyclic Pyridines Containing a Ring-Junction Nitrogen," Green Chemistry, Vol. 12, No. 11, 2010, pp. 2043-2052. doi:10.1039/c0gc00373e

[34] J. Zhang, Z. Cui, F. Wang, Y. Wang, Z. Miao and R. Chen, "Mannich Type Reactions of Chlorophosphites, Phosphoramides and Aldehydes (Ketones) under Solvent-Free and Catalyst-Free Conditions-Synthesis of NPhosphoramino [Small Alpha]-Aminophosphonates," Green Chemistry, Vol. 9, No. 12, 2007, pp. 1341-1345. doi:10.1039/b710008f

[35] N. Annan, R. Paris and F. Jordan, “(E)-4-(.Alpha.-halop-tolyl)-2-oxo-3-butenoic Acids Inhibit Yeast Pyruvate Decarboxylase by a Diversity of Mechanisms: Multiple Fate for the Thiamin-Bound Enamine Intermediate," Journal of the American Chemical Society, Vol. 111, No. 24, 1989, pp. 8895-8901. doi:10.1021/ja00206a019 